\title{
Salivary adiponectin concentration in healthy adult males in relation to anthropometric measures and fat distribution
}

\author{
${ }^{1,2}$ Abdalla MMI, ${ }^{1}$ Soon SC \\ ${ }^{1}$ Physiology Department, Faculty of Medicine, MAHSA University, Kuala Lumpur, Malaysia; \\ ${ }^{2}$ Physiology Department, Faculty of Medicine, Minia University, Egypt \\ E-mail:dr.mona@mahsa.edu.my
}

Objectives. Body fat content, fat distribution, and adiponectin level are important variables in the development of obesity related complications. Anthropometric indices may provide an economic and faster method in measuring the risk for complications through their predictive effect of fat distribution and adiponectin concentration. We aimed to determine, which of the waist circumference (WC), waist-to-hip ratio (WHR), and body mass index (BMI) may be the best predictor for the total fat percentage (WF), visceral fat level (VF), and subcutaneous whole-body fat (SCWBF). We aimed also to investigate the potential use of the anthropometric measures and fat distribution as predictors for the salivary adiponectin level in the healthy adult males.

Subjects. A total of 88 adult males aged between 18-25 years with a wide range of BMI were studied. Anthropometric indices were measured using standardized methods and salivary adiponectin level was assessed using an enzyme-linked immunosorbent assay.

Results. In path analysis of the Structural Equation Model (SEM) using IBM@SPSS AMOS, version22, BMI and WC, but not WHR, were strong predictors for WF and SCWBF $(\mathrm{p}<0.05)$. $\mathrm{BMI}$ but not WC was a strong predictor for $\mathrm{VF}(\mathrm{p}<0.001)$. WF was strong predictor for SCWBF $(\mathrm{p}<0.001)$, but not for VF. BMI, WC, WHR, WF, VF, and SCWBF were poor predictors of the salivary adiponectin level.

Conclusion. BMI is the best predictor for the total body fat and fat distribution. However, WHR seems to be of a little value and the salivary adiponectin level independent of BMI and body fat in healthy adult Malay males.

Key words: adiponectin, body mass index, waist-to-hip ratio, visceral fat, subcutaneous fat, structural equation modeling

The obesity has become a major worldwide health problem that is increasing in prevalence in both developed and developing countries. The dramatic increase in the prevalence of obesity has been reported to be associated with the increase in the prevalence of chronic diseases. The excessive fat deposition in obese individuals has been established to be correlated with the development of metabolic and cardio- vascular complications (Chew et al. 2014). The health consequences associated with obesity differ depending on the place where the adipose tissue is stored; for instance, subcutaneously versus internally (visceral) (Pou et al. 2007; Ducluzeau et al. 2010; Wildman et al. 2011). Although in not all obese individuals of type 2 diabetes or cardiovascular diseases will eventually develop, abdominal adiposity, especially visceral fat,

Corresponding author: Dr. Mona Mohamed Ibrahim Abdalla, Jalan Elmu, Off Jalan University, 59100, Kuala Lumpur, Malaysia; phone: 006-018-2024126; fax: 6037965 2688; e-mail: monaalbetar@yahoo.com; dr.mona@mahsa.edu.my. 
has been found to be a risk for the developing of the metabolic syndrome, insulin resistance, type 2 diabetes mellitus, and cardiovascular diseases (McLaughlin et al. 2011; Neeland et al. 2013).

In addition to the regional fat distribution, as being accountable for variations in the development of obesity-related complications, adiponectin (an adipokine released from the adipose tissue) has been reported to be also involved in the pathogenesis of obesity and insulin resistance (Gao et al. 2012). Results of some studies have indicated a positive correlation between the circulating adiponectin levels and the insulin sensitivity (Goropashnaya et al. 2009; Gao et al. 2012; Vega and Grundy 2013). Lower plasma adiponectin concentrations have been detected in obese patients and patients with insulin resistance when compared to the healthy non-obese individuals. Therefore, low adiponectin levels have been reported to be associated with an increased risk of the development of type 2 diabetes (Vega and Grundy 2013).

Accurate measurement of the compartmental fat distribution using imaging techniques, as magnetic resonance imaging (MRI) and computed tomography (CT) (Lee et al. 2015), are relatively expensive and complex. The adiponectin level assay which uses standardized method is also expensive, especially in the clinical risk assessments among large groups of population and large-scale studies. Simple clinical anthropometric measures, such as BMI, WC, and WHR, can be good alternative methods to assess the risk of developing obesity-related complications in the case that they are proven to be good predictors for fat and compartmental fat distribution; visceral versus subcutaneous, as well as adiponectin level.

There are conflicting findings regarding the possible influence of BMI and body fat on the level of adiponectin. Some studies have reported a significant association between the adiponectin level with BMI and the fat distribution (Staiger et al. 2003; Goropashnaya et al. 2009). However, a lack of association of adiponectin with BMI has been also reported by others (Kuo and Halpern 2011). These have become an important reason to carry out this study by using a statistical tool to elucidate this predicament.

The saliva has recently become an attractive diagnostic tool that can be used as an alternative tool for the measure of blood biomarkers because of its noninvasive sampling method and the good correlation between the protein levels of saliva and plasma (Desai and Mathews 2014). Assays are now available to analyze various parameters in saliva including the measurement of the salivary adiponectin. Many studies have reported a successful measurement of adiponec- tin in the saliva of healthy individuals and diabetic patients (Toda et al. 2007; Thanakun et al. 2013).

Various Asian populations have been reported to have high percentage of body fat even with low BMI (Wang et al. 1994) suggesting that even with low or normal BMI, there is still a risk of the cardiovascular diseases and metabolic syndrome development. To rule out other confounding race-related effects, this study hereby focuses only on the Malay population. To the best of our knowledge, no previous studies have analyzed the possible predictive effect of anthropometric measures, visceral fat, and subcutaneous fat on the salivary adiponectin level in the healthy adult Malay males.

The present study is aimed to determine the relative contribution of WC, WHR, and BMI in predicting the total body fat and its distribution (visceral and subcutaneous fat) and the potential use of these anthropometric measures (WC, WHR, and BMI) and fat distribution as salivary adiponectin level predictors in the healthy adult males via path analysis of a structural equation modeling that is constructed and statistically tested for fitness with the saturated perfect model using IBM@SPSSAMOS, version22.

\section{Materials and Methods}

Ethical clearance and study population. The study design and consent forms were approved by Research Management Committee of MAHSA University, Kuala Lumpur, Malaysia. All procedures performed in studies involving human participants were in accordance with the ethical standards of the institutional and/or national research committee and with the 1964 Helsinki declaration and its later amendments or comparable ethical standards. A total of 88 healthy adult Malay males aged between 18-25 years old participated in the study. All the participants were fully informed about the objectives of the study before completing their written consent forms. Subjects with history of diabetes or cardiac diseases were excluded from the study.

Anthropometric measurements. The height of each subject was measured to the nearest $0.1 \mathrm{~cm}$ using the stadiometer. Body weight, BMI, whole fat \%, visceral fat level, and whole body subcutaneous fat were measured by Omron Karada Scan HBF-362 Bioelectrical impedance. This bioimpedance method of measuring fats has been proven to correlate well with body fat analyzed by Dual-energy X-ray absorptiometry (DEXA) (Fakhrawi et al. 2009; Sluyter et al. 2010) and even by CT (Lee et al. 2015). Each subject was asked to stand barefooted and upright in the middle 
of the sensor platform and hold the fat analyzer with his extended arms. Waist and hip circumferences of the subjects were measured to the nearest $0.1 \mathrm{~cm}$ using measuring tape on standing. Waist circumference was measured by placing the measuring tape placed around the waist at midpoint between the lower costal border (10th rib) and the iliac crest at the end of normal expiration. Hip circumference was measured at the maximum circumference over the buttocks. Then the WHR was calculated (Cameron et al. 2012).

Saliva collection. Non-stimulated saliva of an average of $5 \mathrm{ml}$ was collected using the drooling technique from each subject. All subjects were advised not to eat or drink for at least one hour before saliva collection. They were given proper instructions to ensure collection of suitable and uncontaminated saliva samples and were advised to rinse their mouths with water thoroughly before saliva collection. Over duration of three weeks, saliva samples were collected into test tubes between 9:00 a.m. and 12:00 noon, and kept in ice then centrifuged for $15 \mathrm{~min}$ at $1000 \mathrm{rpm}$ at $4^{\circ} \mathrm{C}$. The supernatant was then divided into aliquots of $1 \mathrm{ml}$ in Eppendorf tubes and stored at $-80^{\circ} \mathrm{C}$ till analysis (Thanakun et al. 2013).

Assay for the salivary adiponectin level. The saliva samples were brought to room temperature and adiponectin was measured by enzyme-linked immunosorbent assay (ELISA). The commercial ELISA kit for human adiponectin (Abnova KA3019) was used. The assay was conducted according to the manufacturer's instructions. Saliva samples were analyzed without dilution. The standards and the samples were measured each in duplicates.

Statistical analysis. Data were analyzed using a structural equation modeling in IBM@SPSS AMOS version 22 with path analysis (Hoyle 1995; Bardenheier et al. 2013; Lee et al. 2016). A model of 7 observed variables (BMI, WC, WHR, WF, VF, SCWBF, and adiponectin) was structured where BMI, WC and WHR represented independent variables for predicting WF, VF, SCWBF, whereas the total fat percentage was an independent variable for predicting $\mathrm{VF}$ and SCWBF. BMI, WC, WHR, WF, VF, SCWBF were independent variables for predicting salivary adiponectin level. This structural equation modeling is advantageous over the traditional regression analysis because of its ability to test the direct and indirect effects of multiple independent variables on dependent variables simultaneously, as well as the associations amongst the independent variables (Kline 2010). A direct effect is represented as an arrow emerging from an independent variable and pointing to an outcome (dependent variable) whereas an indirect effect is represented by the presence of a mediating variable in between the independent and dependent variables. The mediator has an arrow pointing to it from an independent variable and another arrow pointing on to yet another dependent variable. A two-headed arrow indicates the correlation between two variables. The hypothesized model was then assessed for its statistical fit to the data using standardized criteria including chi-square, degree of freedom, probability level, root mean residual (RMR), goodness of fit index (GFI), normed fit index (NFI), incremental fit index (IFI), Tucker-Lewis Index (TLI), comparative fit index (CFI) and the root mean square error of approximation (RMSEA) (Hoyle 1995; Schumacker and Lomax 2004). Modification indices were used to assess specific paths for best fit model. Lastly, model fit statistics, correlations between BMI, WC and WHR as independent variables, squared regression analysis and the standardized regression weights estimates (direct, indirect and total) of anthropometric measurements, total fat and fat distribution on the salivary adiponectin level were reported. All paths with $\mathrm{p}<0.05$ are considered significant.

\section{Results}

Characteristics of the participants. Table 1 shows the means of the anthropometric characteristics, VF level, SCWBF, WF, and the salivary adiponectin level of the participants. In average, the subjects were young adults with wide range of BMI, mainly overweight and pre-obese with high WF according to the World Health Organization (WHO) classification (WHO 2004). However, their average VF level is found to be of 8.59 that is equivalent to $80.59 \mathrm{~cm}^{2}$, which is lower than $100 \mathrm{~cm}^{2}$; the VF threshold for increased risk (Nicklas et al. 2003; Pickhardt et al. 2012) as well as the mean of their WC is considered normal for this age group (Lean et al. 1995).

Fitness of the final structural equation model. Table 2 shows the fit measures of the final structural equation model shown in Figure 1. The fit measures indicate that our model is not different from the saturated model or the perfect model that fit our data. This indicates that the model has correctly described the relations between the measured variables. However, researcher can still modify the model to fit different data of different population until a better fit model is obtained.

Results of path analysis of the model.

BMI, WC and WHR as predictors of WF, VF, and SCWBF. The analysis revealed a strong positive correlation between the three anthropometric measure- 
Table 1

Anthropometric characteristics, visceral fat, subcutaneous whole-body fat and salivary adiponectin levels of the 88 subjects

\begin{tabular}{lc}
\hline Parameter & Mean \pm SD \\
\hline Age $($ year $)$ & $20.64 \pm 2.01$ \\
Height $(\mathrm{cm})$ & $168.82 \pm 5.51$ \\
Weight $(\mathrm{kg})$ & $71.80 \pm 20.1$ \\
BMI $\left(\mathrm{kg} / \mathrm{m}^{2}\right)$ & $24.92 \pm 6.55$ \\
WC $(\mathrm{cm})$ & $85.40 \pm 16.46$ \\
HC $(\mathrm{cm})$ & $100.24 \pm 12.48$ \\
WHR & $0.85 \pm 0.07$ \\
WF $(\%$ from body weight $)$ & $20.10 \pm 8.11$ \\
VF $\left({ }^{*}\right)$ & $8.59 \pm 6.71$ \\
SCWB $(\%)$ & $14.39 \pm 6.15$ \\
Salivary adiponectin $(\mathrm{ng} / \mathrm{ml})$ & $17.24 \pm 5.02$ \\
\hline
\end{tabular}

Data are presented as mean \pm SD.

Abbreviations: BMI - body mass index; WC - waist circumference; HC - hip circumference; WHR - waist-to-hip ratio; WF - total fat percentage; VF - visceral fat level; SCWB - subcutaneous whole-body fat. ${ }^{*}$ Visceral fat levels are relative and not absolute values. Visceral fat area $\left(0\right.$ - approx. $\left.300 \mathrm{~cm}^{2}\right)$ are distributed over 30 -unit levels; one-unit level $=10 \mathrm{~cm}^{2}$.

Table 2

Model fit measurements

\begin{tabular}{lc}
\hline Model & Default model \\
\hline Chi-square & 0.853 \\
Degree of freedom & 2 \\
Probability level & 0.653 \\
RMR & 0.005 \\
GFI & 1.00 \\
NFI & 0.999 \\
IFI & 1.001 \\
TLI & 1.009 \\
CFI & 1.00 \\
RMSEA & 0.000 \\
\hline
\end{tabular}

Abbreviations: RMR - Root Mean Residual, zero represents a perfect fit; GFI - Goodness of Fit Index, values that approach 1 indicate acceptable fit; NFI - Normed Fit Index, 1 is ideal; IFI - Incremental Fit Index, values that exceed .90 are regarded as acceptable; TLI - Tucker-Lewis Index, values over 0.90 or over 0.95 are considered acceptable; CFI - Comparative Fit Index exceeds 0.93; RMSEA - Root Mean Square Error of Approximation, a zero value indicates perfect model fit.

ments (BMI, WC and WHR; $\mathrm{p}<0.05$ ), the highest correlation was seen between BMI and WC $(r=0.96)$. WC and BMI but not WHR were strong predictors for $\mathrm{WF}(\mathrm{p}<0.05)$, only the BMI was a strong predictor for
Table 3

Squared multiple correlations for the predictors of total body fat, visceral fat, subcutaneous whole-body fat and salivary adiponectin level

\begin{tabular}{lcccc}
\hline Parameter & $\mathbf{R}^{2}$ & Lower & Upper & p-value \\
\hline WF & $0.831^{\star}$ & 0.731 & 0.886 & 0.004 \\
SCWBF & $0.987^{\star}$ & 0.970 & 1.000 & 0.001 \\
VF & $0.992^{\star}$ & 0.987 & 0.995 & 0.003 \\
$\begin{array}{l}\text { Salivary } \\
\text { adiponectin }\end{array}$ & 0.043 & - & 0.066 & 0.467 \\
\hline
\end{tabular}

WF - total body fat (\%); SCWBF - subcutaneous whole-body fat; $V F$ - visceral fat. ${ }^{*} \mathrm{p}<0.05$

VF (when BMI goes up by 1 standard deviation, VF goes up by 0.93 standard deviations), BMI and WC were good predictors for SCWBF $(\mathrm{p}<0.05)$. For WF, as a predictor for SCWBF versus VF, it seemed to be a strong predictor for SCWBF but not $\mathrm{VF}(\mathrm{p}<0.001$ and 0.74 , respectively) (when WF goes up by 1 standard deviation, SCWBF goes up by 0.763 standard deviations) (Figure 1). It is estimated that the predictors of WF could explain $83.1 \%$ of its variance, the predictors of SCWB could explain $98.7 \%$ of its variance and the predictors of VF could explain $99.2 \%$ of its variance as indicated by the squared multiple correlations estimates $\left(\mathrm{R}^{2}\right)$ (Table 3$)$.

The direct, indirect and total predictive effects of the anthropometric and body fat mass measurements on the salivary adiponectin level. The analysis showed that the anthropometric measurements were poor predictors for the salivary adiponectin level directly, indirectly (mediated by their effects on WF, VF, and SCWBF) and total effects of both direct and indirect (Table 4). It is estimated that the predictors for the salivary adiponectin level could explain only $4.3 \%$ of its variance. In other words, the error variance of adiponectin is approximately $95.7 \%$ of the variance of adiponectin itself (Table 3 ).

\section{Discussion}

The correlation and regression analysis suggest that in healthy young adult Malay males, who are on average overweight-to-obese, WC and BMI, but not WHR, are good predictors for WF. WC and BMI were not significantly different in predicting the SCWBF. Many studies have investigated the association of the anthropometric measures with body fat percentage and its distribution (Goulding et al. 1998; Steinberger et al. 2005; Suchanek et al. 2012; Ejtahed et al. 2014). Suchanek et al. (2012) have reported 
Table 4

Standardized regression estimates of direct, indirect and total effect of body mass index, waist circumference, waist-to-hip ratio, total body fat, subcutaneous whole-body fat, and visceral fat on salivary adiponectin level

\begin{tabular}{|c|c|c|c|c|c|c|}
\hline & WHR & WC & BMI & WF & SCWB & VF \\
\hline \multicolumn{7}{|c|}{ Standardized total effects } \\
\hline WF & 0.014 & 0.483 & $0.426^{*}$ & - & - & - \\
\hline SCWB & 0.010 & $0.483^{*}$ & $0.461^{\star}$ & $0.763^{*}$ & - & - \\
\hline VF & 0.000 & 0.061 & $0.937^{*}$ & 0.017 & - & - \\
\hline Salivary adiponectin & -0.063 & -0.170 & 0.108 & 0.062 & -0.348 & 1.861 \\
\hline \multicolumn{7}{|c|}{ Standardized direct effects } \\
\hline WF & 0.014 & 0.483 & $0.426^{*}$ & - & - & - \\
\hline SCWB & - & $0.114^{*}$ & $0.136^{*}$ & $0.763^{*}$ & - & - \\
\hline VF & - & 0.052 & $0.930^{*}$ & 0.017 & - & - \\
\hline Salivary adiponectin & -0.064 & -0.258 & -1.602 & 0.296 & -0.348 & 1.861 \\
\hline \multicolumn{7}{|c|}{ Standardized indirect effects } \\
\hline WF & - & - & - & - & - & - \\
\hline SCWB & 0.010 & 0.368 & $0.325^{*}$ & - & - & - \\
\hline VF & - & 0.008 & 0.007 & - & - & - \\
\hline Salivary adiponectin & 0.001 & 0.088 & 1.710 & -0.234 & - & - \\
\hline
\end{tabular}

Abbreviations: BMI - body mass index; WC - waist circumference; WHR - waist-to-hip ratio; WF - total body fat (\%); VF - visceral fat; SCWBF - subcutaneous whole-body fat. ${ }^{*} \mathrm{p}<0.05$

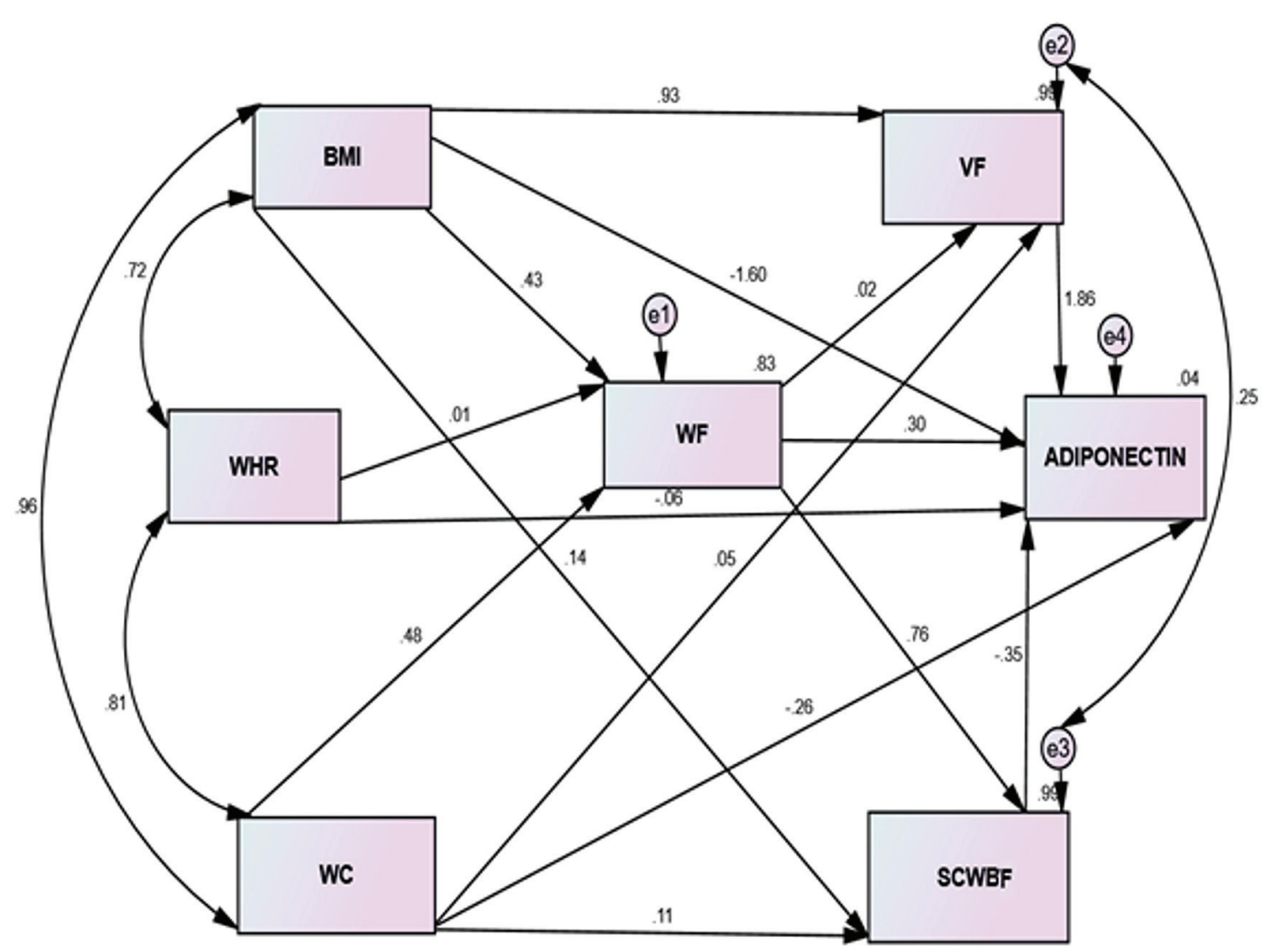

Figure 1. The final Structural Equation Model (SEM) with standardized estimates. 
a poor predictive effect of WHR on total body fat in Caucasians and similar findings have been reported among Iranian adolescents by Ejtahed et al. (2014). The poor effect of WHR in predicting WF found is supported by studies on the French-Canadian males and females with an age range from 18-72 years (Rankinen et al. 1999). Similarly, a study being done on weight loss evaluation in obese males and females have reported that WHR is not appropriate for evaluating the changes in the visceral fat depot (van der Kooy et al. 1993).

The body mass index rather than WC predicted VF. This poor predictive effect of WC for VF is contradictory to the findings of Rankinen et al. (1999) about WC being the best predictor for VF. This can be explained by the difference in the race used as body fat distribution is affected by race.

The waist circumference reflects not only the intraabdominal but also the abdominal subcutaneous fat, concluding that in Malay adult males who are overweight, the fat is rather accumulated subcutaneously than viscerally. This is evidenced in our study by the strong positive predictive effect of the total fat percentage on the subcutaneous body but not the visceral fat. These findings strengthen the results of a similar study done on healthy adults (Chen et al. 2014). However, they were different from a study done on children and reported a significant association of WF with both the visceral and the subcutaneous fat (Saelens et al. 2007). This difference can be explained by the different age groups used.

The analysis of the structural equation model revealed that parameters such as BMI, WC, WHR, $\mathrm{WF}, \mathrm{VF}$, and SCWBF, each or in combination, are not suitable to use as predictors for the salivary level of adiponectin in our study population as evidenced by the insignificant direct, indirect, and total effects of each of these anthropometrics on the adiponectin (as shown in Figure 1 and Table 4) and the low $\mathrm{R}^{2}$ of their total effect on adiponectin (shown in Table 3). This is consistent with the conclusion drawn from a meta-analysis study of Kuo and Halpern (2011). They have performed analysis of several obesity studies and plasma adiponectin levels in healthy adults and rejected a hypothesis that obesity may independently affect plasma adiponectin. The insignificant negative effect of WHR, SCWBF, and WC in predicting adiponectin concentration (shown in Table 3) is in agreement with the study being done to investigate the relationship of serum adiponectin concentrations with body fat distribution which re- ported a negative correlation between WHR as well as WC and adiponectin (Staiger et al. 2003). Our findings indicate that BMI, WF, VF, and SCWBF are poor predictors for the considering of the salivary adiponectin concentrations in the healthy adult males. This supports the conclusions drawn from other studies on the tight physiological regulation of adiponectin concentration that is not essentially affected by just an expansion of the body fat. This has been evidenced in some obese individuals who remained healthy due to their higher capacity to hold storage fat because of genetic or environmental factors (Virtue and Vidal-Puig 2010). It has also been observed that adiponectin resistance had developed earlier than insulin resistance following a high fat diet in mice (Mullen et al. 2009) reflecting the regulation of adiponectin by feedback mechanism. Hypoadiponectinemia has been found to be associated with the obesity related pathological conditions such as metabolic syndrome (Liu and Liu 2009) that is considered as a condition of tissue inflammation (Gustafson et al. 2007). In addition to the presence of high plasma glucose, fat intake, and dyslipidemia with metabolic syndrome, are all known to inhibit adiponectin secretion in vitro (Oller do Nascimento et al. 2009; Sun et al. 2009; Lapointe et al. 2011).

\section{Conclusions}

Our study declassified the interrelations of anthropometric indices in tandem with the levels of the salivary adiponectin as detected in a group of subjects with a wide range of BMI. In the healthy young adult Malay males, BMI and WC, but not WHR, showed up as strong predictors for the changes in WF and SCWBF. BMI, but not WC, can be used as a predictor of VF. However, the salivary adiponectin level is independent on BMI and body fat.

\section{Acknowledgements}

We would like to thank MAHSA Research Management Centre for funding this research and giving us the opportunity to conduct this study at MAHSA University. We would like to thank all the participants who agreed to take part in our study, Faculty of Medicine at MAHSA University for providing the facilities needed, and Dr. Mahmoud Said Mohdy for his help in the statistics and analysis. This study was funded by MAHSA University (grant number RP 7210/14). 


\section{References}

Bardenheier BH, Bullard KM, Caspersen CJ, Cheng YJ, Gregg EW, Geiss LS. A novel use of structural equation models to examine factors associated with prediabetes among adults aged 50 years and older: National Health and Nutrition Examination Survey 2001-2006. Diabetes Care 36, 2655-2662, 2013.

Cameron AJ, Magliano DJ, Shaw JE, Zimmet PZ, Carstensen B, Alberti KG, Tuomilehto J, Barr EL, Pauvaday VK, Kowlessur S, Soderberg S. The influence of hip circumference on the relationship between abdominal obesity and mortality. Int J Epidemiol 41, 484-494, 2012.

Chen CH, Chen YY, Chuang CL, Chiang LM, Chiao SM, Hsieh KC. The study of anthropometric estimates in the visceral fat of healthy individuals. Nutr J, 13-46, 2014.

Chew WF, Masyita M, Leong PP, Boo NY, Zin T, Choo KB, Yap SF. Prevalence of obesity and its associated risk factors among Chinese adults in a Malaysian suburban village. Singapore Medic J 55, 84-91, 2014.

Desai GS, Mathews ST. Saliva as a non-invasive diagnostic tool for inflammation and insulin-resistance. World J Diabetes 5, 730-738, 2014.

Ducluzeau PH, Manchec-Poilblanc P, Roullier V, Cesbron E, Lebigot J, Bertrais S, Aube C. Distribution of abdominal adipose tissue as a predictor of hepatic steatosis assessed by MRI. Clin Radiol 65, 695-700, 2010.

Ejtahed HS, Asghari G, Mirmiran P, Hosseinpour-Niazi S, Sherafat-KazemZadeh R, Azizi F. Body mass index as a measure of percentage body fat prediction and excess adiposity diagnosis among Iranian adolescents. Arch Iran Med 17, 400-405, 2014.

Fakhrawi DH, Beeson L, Libanati C, Feleke D, Kim H, Quansah A, Darnell A, Lammi-Keefe CJ, Cordero-MacIntyre Z. Comparison of body composition by bioelectrical impedance and dual-energy x-ray absorptiometry in overweight/obese postmenopausal women. J Clin Densitom 12, 238-44, 2009.

Gao H, Salim A, Lee J, Tai ES, van Dam RM. Can body fat distribution, adiponectin levels and inflammation explain differences in insulin resistance between ethnic Chinese, Malays and Asian Indians? Int J Obes [Lond] 36, 1086-1093, 2012.

Goropashnaya AV, Herron J, Sexton M, Havel PJ, Stanhope KL, Plaetke R, Mohatt GV, Boyer BB. Relationships between plasma adiponectin and body fat distribution, insulin sensitivity, and plasma lipoproteins in Alaskan Yup'ik Eskimos: The Center for Alaska Native Health Research study. Metabolism 58, 22-29, 2009.

Goulding A, Gold E, Cannan R, Taylor RW, Williams S, Lewis-Barned NJ. DEXA supports the use of BMI as a measure of fatness in young girls. Int J Obes 20, 1014-1021, 1998.

Gustafson B, Hammarstedt A, Andersson CX, Smith U. Inflamed adipose tissue: a culprit underlying the metabolic syndrome and atherosclerosis. Arterioscler Thromb Vasc Biol 27, 2276-2283, 2007.

Hoyle R. Structural equation modelling: concepts, issues and applications. Thousand Oaks, CA: Sage Publications, 1995.

Kline RB. Principles and Practice of Structural Equation Modeling. 3rd ed. New York, NY, The Guilford Press, 2010.

Kuo SM, Halpern MM. Lack of association between body mass index and plasma adiponectin levels in healthy adults. Int J Obes [Lond] 35, 1487-1494, 2011.

Lapointe A, Tchernof A, Lamarche B, Piche ME, Weisnagel J, Bergeron J, Lemieux S. Plasma adiponectin concentration is strongly associated with VLDL-TG catabolism in postmenopausal women. Nutr Metab Cardiovasc Dis 21, 254-260, 2011.

Lean ME, Han TS, Morrison CE. Waist circumference as a measure for indicating need for weight management. BMJ 311, 158-61, 1995.

Lee DH, Park KS, Ahn S, Ku EJ, Jung KY, Kim YJ, Kim KM, Moon JH, Choi SH, Park KS1, Jang HC, Lim S. Comparison of Abdominal Visceral Adipose Tissue Area Measured by Computed Tomography with That Estimated by Bioelectrical Impedance Analysis Method in Korean Subjects. Nutrients 7, 10513-10524, 2015.

Lee EH, Lee YW, Moon SH. A Structural Equation Model Linking Health Literacy to Self-efficacy, Self-care Activities, and Health related Quality of Life in Patients with Type 2 Diabetes. Asian Nurs Res (Korean Soc Nurs Sci), 10, 82-7, 2016.

Liu M, Liu F. Transcriptional and post-translational regulation of adiponectin. Biochem J 425, 41-52, 2009.

McLaughlin T, Lamendola C, Liu A, Abbasi F. Preferential fat deposition in subcutaneous versus visceral depots is associated with insulin sensitivity. J Clin Endocrinol Metabol 96, E1756-E1760, 2011.

Mullen KL, Pritchard J, Ritchie I, Snook LA, Chabowski A, Bonen A, Wright D, Dyck DJ. Adiponectin resistance precedes the accumulation of skeletal muscle lipids and insulin resistance in high-fat-fed rats. Am J Physiol Regul Integr Comp Physiol 296, R243-R251, 2009. 
Neeland IJ, Ayers CR, Rohatgi AK, Turer AT, Berry JD, Das SR, Vega GL, Khera A, McGuire DK, Grundy SM, de Lemos JA. Associations of visceral and abdominal subcutaneous adipose tissue with markers of cardiac and metabolic risk in obese adults. Obesity [Silver Spring] 21, E439-E447, 2013.

Nicklas BJ, Penninx BW, Ryan AS, Berman DM, Lynch NA, Dennis KE. Visceral adipose tissue cut-offs associated with metabolic risk factors for coronary heart disease in women. Diabetes Care 26, 1413-1420, 2003.

Oller do Nascimento CM, Ribeiro EB, Oyama LM. Metabolism and secretory function of white adipose tissue: effect of dietary fat. An Acad Bras Cienc 81, 453-466, 2009.

Pickhardt PJ, Jee Y, O’Connor SD, del Rio AM. Visceral adiposity and hepatic steatosis at abdominal CT: Association with the metabolic syndrome. AJR Am J Roentgenol 198, 1100-1107, 2012.

Pou KM, Massaro JM, Hoffmann U, Vasan RS, Maurovich-Horvat P, Larson MG, Keaney JF Jr, Meigs JB, Lipinska I, Kathiresan S, Murabito JM, O’Donnell CJ, Benjamin EJ, Fox CS. Visceral and subcutaneous adipose tissue volumes are cross-sectionally related to markers of inflammation and oxidative stress: the Framingham Heart Study. Circulation 116, 1234-1241, 2007.

Rankinen T, Kim SY, Perusse L, Despres JP, Bouchard C. The prediction of abdominal visceral fat level from body composition and anthropometry: ROC analysis. Int J Obes Relat Metab Disord 23,801-819, 1999.

Saelens BE, Seeley RJ, van Schaick K, Donnelly LF, O’Brien KJ. Visceral abdominal fat is correlated with whole-body fat and physical activity among 8-y-old children at risk of obesity. Am J Clin Nutr 85, 46-53, 2007.

Schumacker RE, Lomax RG. A beginner's guide to structural equation modeling, Second edition. Mahwah, NJ: Lawrence Erlbaum Associates 2004.

Sluyter JD, Schaaf D, Scragg RK, Plank LD. Prediction of fatness by standing 8-electrode bioimpedance: a multiethnic adolescent population. Obesity [Silver Spring] 18, 183-189, 2010.

Staiger H, Tschritter O, Machann J, Thamer C, Fritsche A, Maerker E, Schick F, Haring HU, Stumvoll M. Relationship of serum adiponectin and leptin concentrations with body fat distribution in humans. Obes Res 11, 368-372, 2003.

Steinberger J, Jacobs DR, Raatz S, Moran A, Hong CP, Sinaiko AR. Comparison of body fatness measurements by $\mathrm{BMI}$ and skinfolds vs dual energy X-ray absorptiometry and their relation to cardiovascular risk factors in adolescents. Int J Obes [Lond] 29, 1346-1352, 2005.

Suchanek P, Kralova Lesna I, Mengerova O, Mrazkova J, Lanska V, Stavek P. Which index best correlates with body fat mass: BAI, BMI, waist or WHR? Neuro Endocrinol Lett 2, 78-82, 2012.

Sun J, Xu Y, Dai Z, Sun Y. Intermittent high glucose stimulates MCP-1, IL-18, and PAI-1, but inhibit adiponectin expression and secretion in adipocytes dependent of ROS. Cell Biochem Biophys 55,173-180, 2009.

Thanakun S, Watanabe H, Thaweboon S, Izumi Y. An effective technique for the processing of saliva for the analysis of leptin and adiponectin. Peptides 47, 60-65, 2013.

Toda M, Tsukinoki R, Morimoto K. Measurement of salivary adiponectin levels. Acta Diabetol 44, 20-22, 2007.

van der Kooy K, Leenen R, Seidell JC, Deurenberg P, Droop A, Bakker CJ. Waist-hip ratio is a poor predictor of changes in visceral fat. Am J Clin Nutr 57, 327-33, 1993.

Vega GL and Grundy SM. Metabolic risk susceptibility in men is partially related to adiponectin/leptin ratio. J Obes 2013, 409679, 2013.

Virtue S, Vidal-Puig A. Adipose tissue expandability, lipotoxicity and the metabolic syndrome Fan allostatic perspective. Biochim Biophys Acta 1801, 338-349, 2010.

Wang J, Thornton JC, Russell M, Burastero S, Heymsfield S, Pierson RN Jr. Asians have lower body mass index [BMI] but higher percent body fat than do whites: comparisons of anthropometric measurements. A J Clin Nutr 60, 23-28, 1994.

WHO Expert Consultation. Appropriate body mass index for Asian populations and its implication for policy and intervention strategies. Lancet 363, 157-163, 2004.

Wildman RP, Janssen I, Khan UI, Thurston R, Barinas-Mitchell E, El Khoudary SR, Everson-Rose SA, Kazlauskaite R, Matthews KA, Sutton-Tyrrell K. Subcutaneous adipose tissue in relation to subclinical atherosclerosis and cardiometabolic risk factors in midlife women. A J Clin Nutr 93, 719-726, 2011. 